\title{
Research on Active Battery Management of Substation DC Power Supply
}

\author{
Lei Bo $^{1, ~ a ~}$, Lu Zhigang ${ }^{1, b}$, Zheng Yongqiang ${ }^{2, c}$, Ji Qinghong ${ }^{2, d}$ \\ ${ }^{1}$ State Key Laboratory of HVDC, Electric Power Research Institute, China Southern Power Grid, \\ Guangzhou, 510080, China \\ ${ }^{2}$ Zhuhai Watt Electric Equipment Co., LTD, Zhuhai, 519080,China \\ aemail: leibo@csg.cn, bemail: \\ luzg@csg.cn, cemail:watt_KY@163.com, demail:jiqinghong@watt.com
}

Keywords: Substation; battery; active battery management

\begin{abstract}
Based on an introduction on present status of battery management of substations in China, this paper points out problems existing in current battery management of substation, puts forward an active battery management strategy regarding such problems and introduces its implementation framework, principle, the technical characteristics and significance
\end{abstract}

\section{Introduction}

As an important part of substation DC power, the battery serves to provide the required transient high current to the feeder switch and relay protection actions as well as the back-up power supply when the substation loses AC power to maintain the uninterrupted operation of the overall secondary system. The normal performance of battery is crucial for the stable operation of overall transformer substation. At present, by employing the charger for battery charging management, and the battery voltage testing device for testing of single battery voltage, the substation DC power supply systems can realize the equalized battery charging management, battery pack voltage alarm, single battery voltage alarm and other basic functions. Simple testing of single battery voltage can't accurately and timely reflect the status of battery, such as the increase in single battery resistance, increase in contact resistance of connecting lines, decrease in battery capacity, open circuit inside battery, etc., making it difficult to find potential problems of battery in daily operation. For the same reason, the battery often has caused serious loss to the operation of substation or even the distribution network when the failure occurs[1]. Typical substation accidents caused by such failure in China are shown in table 1.

Table 1: Typical substation accidents

\begin{tabular}{ccc}
\hline No. & Accident & Cause \\
\hline 1 & "4.29" Fuizhou Lanba Station - 2013 & Multi-battery open circuit \\
2 & "10.7" Yangjiang Shiwan Station - 2015 & Inaccurate capacity testing of battery \\
3 & "8.18" Xi'an Nanjiao Station - 2016 & Disconnection of battery from bus \\
\hline
\end{tabular}

\section{Present Status of Battery management}

According to O\&M regulations of State Grid Corporation of China and China Southern Power Grid, the substation O\&M personnel are required to conduct the battery capacity test on a regular basis. However, due to the large scale of current power grid, great number of substations as well as the long period of and safety risks in battery discharging and recharging, manual maintenance and capacity testing requires abundant manpower and material investment, which, despite of such investment, will still have batteries in some substations untested in a timely manner in practice. Therefore, it is important and urgent to find an effective way of battery maintenance management.

At present, the main methods of battery management in China are as follows[2].

a) Battery voltage.

It means testing the single battery voltage, judging whether overvoltage or undervoltage occurs, 
and identifying the battery with large voltage deviation, that is, the failed battery (in terms of inconsistency) based on comparison within the pack. The method is easy and has a low cost. However, the floating charge voltage or equalized charge voltage, clamped by the charger, has little relevance with the capacity, and thus cannot directly reflect the battery capacity and performance. Therefore, the method is limited. In addition, most of existing battery voltage detection devices employ the single-cable voltage acquisition method, where a battery shares the same cable with the previous battery, that is, $n$ batteries will have $n+1$ cables, which will eventually result in that the voltage acquisition of the single battery will have superposed voltage drop of cables. The deviation is more obvious during charging or discharging.

b) Battery internal resistance.

It means testing the battery internal resistance with the DC discharge or AC injection method and further determining the battery capacity. The results acquired with different test methods and test equipment lack consistency and repeatability and thus it is difficult to formulate a uniform standard, but the method has certain advantages in identifying the failed battery. In practice, judgment will be made based on the analysis on the trend of test results, that is, testing the battery capacity every once in a while using the same measurement tool, after which the battery internal resistance is measured, recorded and compared with the past values recorded to analyze the battery performance trend based on the change in internal resistance. The method is complex and requires long period of measurement and thus cannot be conducted in real time like voltage acquisition. As a way of passive detection, internal resistance detection can only be used for detection and cannot help to prevent the occurrence of failures.

c) Battery balancing.

It means discharging the single battery forcibly based on the setting of its floating charge voltage to maintain the voltage consistence within the battery pack. Due to inconsistence of batteries, battery matching at the factory and matching accuracy, the performance change of battery in the long period, it is impossible to keep consistent performance among all batteries within the same substation battery pack. The battery balancing method, which conducts forced balancing only based on the floating charge voltage of the single battery, not only fails to identify the internal failure, but also may even artificially enlarge the inconsistency within the pack, causing secondary damage to the battery.

All of the above three methods have drawbacks, which can be summarized into lack of detection methods, delayed detection of resistance and lack of effective ways of increasing battery life.

\section{Principles of Active Battery Management}

There are three main factors affecting the service life of substation DC power batteries: ambient temperature, floating charge voltage and corrosion[3]-[4].

The substation battery serves as the backup power supply and is under the floating charge state in most time. According to current technical regulations on operation and maintenance of grid storage battery DC power in China, the battery requires equalized charging every 3-6 months. The calculation based on the failure-free state shows that battery is under the floating charge state in more than $99 \%$ of the time. Assuming that 2 times of equalized charging are conducted a year, the times of deep charge and discharge of a 6-year battery pack will be just 12 and not go over 20 whatsoever. At present, substations in China have fairly complete infrastructure and can basically meet the requirements in temperature, floating charge voltage and temperature compensation, but fail to conduct sufficient activation management on battery judging from the charge and discharge times in the whole life cycle. Under the long-term floating charging state, the battery will have chemical reactions inside, such as constant increase in sulfuric acid concentration of lead-acid battery, consumption of water and corrosion of negative plate lead by sulfuric acid, which will cause irreversible damage to the battery. Appropriate and timely discharge in the process of floating charge can effectively activate the battery and prolong its service life. Assuming that 1 to 2 times of shallow activation cycles are conducted a month, a ten-year battery will have just 250 cycles, which is within the time of cycles on one hand, and can extend battery life on the other hand. 
Under such background, this paper puts forward active battery management strategy, under which shallow discharge will be conducted with coordinated use of the charger or active load on a regular basis and then the battery will be recharged by the charger automatically. During the process of discharging and recharging, various parameters will be detected, such as the battery voltage, voltage jump, temperature, etc., to acquire various data reflecting battery performance, and further analyze the performance of battery pack and the single battery. Finally, it can realize fairly comprehensive battery state inspection, failed battery positioning and other functions and prolong the service life of battery. The implementation framework of management strategy is shown in figure 1.

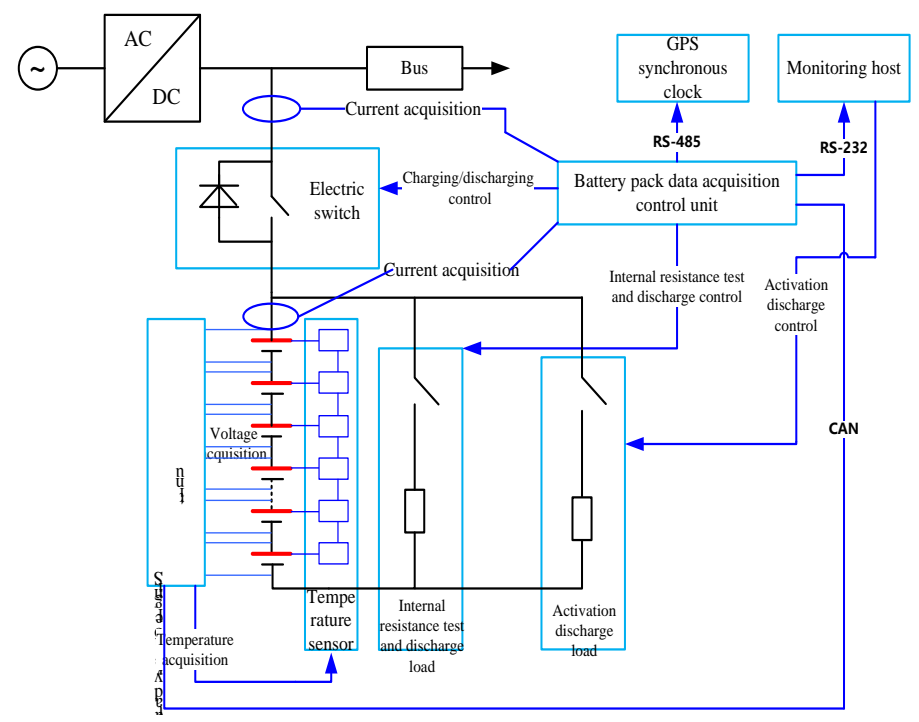

Fig.1. Framework of active battery management strategy

Active battery management mainly has the following technical characteristics:

a) Coordinated management with charger or active load and regular activation. The battery activation will be started automatically or manually based on the activation cycle. During activation, the charger voltage is lowed to the safe voltage of activation to run the battery with full load, or the active load will be employed to discharge and activate the battery without disconnecting the bus. The activation will end automatically when the undervoltage alarm of battery pack or single battery is generated, the activation time set is over, or the level of discharge reaches the setting value. Then, the output voltage of charger will increase to the original floating charge voltage or the active load will be disconnected, after which the charging management system will initiate the recharging process automatically to recharge the battery. The control process is shown in figure 2.

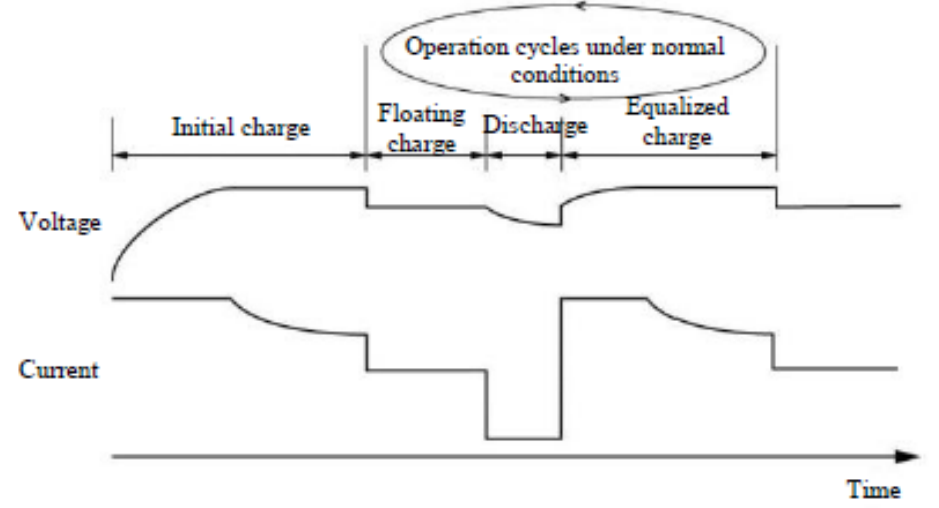

Fig.2. Battery activation control curve

b) Detection and analysis of floating charge current. The detection and analysis of floating charge current can be used to determine the change of battery performance and identify the trend of battery deterioration in advance. If the current change of a single battery is greater than the threshold value, it indicates that the battery has greater internal resistance or faces the risk of performance degradation. Usually the floating charge current of battery is about $1 \mathrm{~mA} / \mathrm{Ah}$. Taking the 300Ah battery for example, the impulse current is $30 \sim 300 \mathrm{~A}$, the charging current is $30 \mathrm{~A}$, and 
the floating charge current is about 0.3A. For such a great range of current, magnetic modulation can effectively isolate and detect weak current, and prevent detection errors caused by electric shock.

c) Voltage jump and performance analysis of single battery. When the battery transits from the floating charge state into activation and discharging, its voltage will get back to the on-load voltage from the floating charge voltage. At the time, the abnormal batteries (such as batteries with large internal resistance, internal open circuit, or insufficient capacity) will have very great voltage jump. The double-wire method (as shown in figure 3) can be used to detect the voltage change in single battery during the activation process, analyze the performance deviation of single battery and finally identify the failed battery.

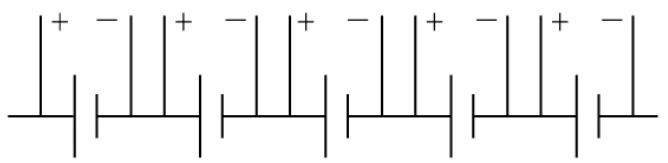

Fig.3. Wiring for single battery voltage acquisition with the double-wire method

d) Temperature measurement and analysis of single battery. The traditional management system usually can only measure the ambient temperature, but the temperature of single battery can more reflect the performance of battery. The battery performance trends can be acquired by using the single bus battery temperature measurement unit to measure the change in single battery temperature, and comparing it with the threshold value. If the range of temperature change is greater than the threshold value, the battery is regarded failed. Also, transverse comparison can be made to track the performance change of batteries with higher charging and discharging temperature in one pack.

e) Battery open circuit analysis. Based on measurement and analysis of the discharge current, voltage jump of single battery when it changes from floating charge to on-load discharge, and the voltage drop of battery pack during discharge, it can be determined whether there is open circuit in the system, to give early warning and reduce the risk of voltage loss of the substation in cases of AC power loss.

\section{Benefits of Active Battery Management}

Active battery management can effectively change the present approach of battery management. Firstly, the activation function can prolong the service life of battery; Secondly, based on the measurement and analysis of current, voltage and temperature during activation, the battery failure can be learned in advance and the failed battery can be positioned to facilitate failure elimination; Finally, the detection results of this system can provide a basis for judging whether to conduct capacity testing and discharge and thus contribute to more targeted capacity test and discharge and higher running and maintenance efficiency.

The active battery management can increase the service life of the battery, lower the risk of voltage loss in DC system due to battery failure and significantly reduce the workload of maintenance personnel.

\section{Conclusion}

The method of substation DC power battery management has been improving all the time, but passive management is still adopted in most cases, which is unable to meet the needs of modern power grid development[5]. Besides anticipation and identification of problems existing in the battery, active management can also prevent the occurrence of battery failure through activation and thus reduce the risk of failure and prolong the service life of the battery. Also, due to the application of the comprehensive detection management, the test data can serve as a reference for battery capacity checking to reduce the workload of maintenance, take targeted measures and improve the operation and maintenance efficiency. 


\section{Acknowledgement}

In this paper, the research was sponsored by the State Key Laboratory of HVDC and Scientific Research Project of Technology Research Center of China Southern Power Grid Co.Ltd (Project No. CSGTRC-142021).

\section{References}

[1] Zhao Xizheng. Strengthen power system security to ensure reliability power delivery[J]. Power System Technology, 2003, 27(10), 1-7.

[2] Xiang Xiaomin, Zhou Baiming, Xu Shamiao. Discussion of on-line monitoring residual capacity of battery[J]. Chinese Journal of Power Sources, 2009, 33(3), 213-216.

[3 WANG Hong, ZHANG Guang-hui, Liang Zhi-qiang, Ran Jian-guo. Networking Management and Condition Based Maintenance of DC Power Supply System in Substations [J]. Power System Technology, 2010, 34(2), 185-189.

[4] Yu Bin. The Research on Intelligent Monitoring System for DC Power Supply [D]. North China Electric Power University, 2013.

[5] CHAI Yu-hong, WANG Run-qin, YANG Ai-sheng. Research and Application of On-line Maintenance Technology for DC System Battery in Substations [J]. Shanxi Electric Power, 2014, 188 (5), 11-13. 